\title{
Existence and Multiplicity of Homoclinic Orbits for Second-Order Hamiltonian Systems with Superquadratic Potential
}

\author{
Ying Lv and Chun-Lei Tang \\ School of Mathematics and Statistics, Southwest University, Chongqing 400715, China \\ Correspondence should be addressed to Chun-Lei Tang; tangcl@swu.edu.cn
}

Received 25 September 2012; Accepted 11 January 2013

Academic Editor: Wenming Zou

Copyright (C) 2013 Y. Lv and C.-L. Tang. This is an open access article distributed under the Creative Commons Attribution License, which permits unrestricted use, distribution, and reproduction in any medium, provided the original work is properly cited.

We investigate the existence and multiplicity of homoclinic orbits for second-order Hamiltonian systems with local superquadratic potential by using the Mountain Pass Theorem and the Fountain Theorem, respectively.

\section{Introduction and Main Result}

Consider the second-order nonautonomous Hamiltonian systems

$$
\ddot{u}(t)-L(t) u(t)+\nabla W(t, u(t))=0,
$$

where $L \in C\left(R, R^{N^{2}}\right)$ is a symmetric matrix valued function, $W \in C^{1}\left(R \times R^{N}, R\right)$, and $\nabla W(t, x)=(\partial W / \partial x)(t, x)$. We say that a nonzero solution $u$ of problem (1) is homoclinic (to 0 ) if $u(t) \rightarrow 0$ and $\dot{u}(t) \rightarrow 0$ as $|t| \rightarrow \infty$.

The existence of homoclinic orbits for Hamiltonian systems and their importance in the study of the behavior of dynamical systems have been already recognized by Poincaré [1]. Only during the last two decades such problem has been studied by using critical point theory.

If $L(t)$ and $W(t, x)$ are independent of $t$ or periodic in $t$, many authors have studied the existence of homoclinic orbits for Hamiltonian systems, see, for instance, [2-9], and a more general case is considered in recent papers $[10,11]$. In this case, the existence of homoclinic orbits is obtained by going to the limit of periodic solutions of approximating problems. In recent years, concentration compactness principle has also been widely used to deal with the perturbations of periodic or autonomous problems, for example, $[12,13]$.

If $L(t)$ and $W(t, x)$ are neither autonomous nor periodic, the problem is quite different from the ones just described, because of the lack of compactness of the Sobolev embedding.
Rabinowitz and Tanaka [14] study without any periodicity assumption and obtain the existence of homoclinic orbits of problem (1) by using a variant of the Mountain Pass Theorem without the Palais-Smale condition under the following condition.

(L) $L \in C\left(R, R^{N^{2}}\right)$ is a symmetric and positively definite matrix for all $t \in R$, and there exists a continuous function $l: R \rightarrow R$ such that $l(t)>0$ for all $t \in R$ and

$$
(L(t) x, x) \geq l(t)|x|^{2}, \quad l(t) \longrightarrow \infty \text { as }|t| \longrightarrow \infty .
$$

Assuming coercivity assumption $(L)$, Omana and Willem [15] obtain an improvement on the latter result by employing a new compact embedding theorem; in fact, they show that the (PS) condition is satisfied and obtain the existence and multiplicity of homoclinic orbits of problem (1) by using the usual Mountain Pass Theorem. After [14] and [15], many results [16-22] are obtained for the case where $L(t)$ is neither constant nor periodic in $t$.

Korman and Lazer [23] remove the technical coercivity in case that $L(t)$ and $W(t, x)$ are even in $t$ and $L(t)$ is positively definite for all $t \in R$, by approximating homoclinic orbits from solutions of boundary value problems, which is complemented by [24].

Most of the papers mentioned previously tackle the superquadratic case (see $[2-10,14-16,18-21,23,24])$ and the subquadratic case (see $[17-19,22,25])$. The following 
Ambrosetti-Rabinowitz condition is widely used in almost all papers tackling the superquadratic case.

(AR) There exists a constant $\mu>2$ such that, for every $t \in R$ and $x \in R^{n} \backslash\{0\}$,

$$
0<\mu W(t, x) \leq(\nabla W(t, x), x) .
$$

Many recent papers have complemented the (AR) condition, for example, [6, 16, 20-22, 24].

There are also many papers that tackle the multiplicity of homoclinic orbits, for example [20, 21, 23-25]. In particular, based on the variant Fountain Theorem of [26], Yang and Han [19] consider the multiplicity of homoclinic orbits for problem (1).

Theorem A (see [19, Theorem 1.2]). Suppose that $L(t)$ satisfies $(L)$ and $\left(L^{\prime}\right)$. For some $a>0$ and $\bar{r}>0$, one of the following is true:

(i) $L \in C^{1}\left(R, R^{N^{2}}\right)$ and $\left|L^{\prime}(t)\right| \leq a|L(t)|$, for all $|t| \geq \bar{r}$, or

(ii) $L \in C^{2}\left(R, R^{N^{2}}\right)$ and $L^{\prime \prime}(t) \leq a L(t)$, for all $|t| \geq \bar{r}$,

where $L^{\prime}(t)=(d / d t) L(t)$ and $L^{\prime \prime}(t)=\left(d^{2} / d t^{2}\right) L(t)$ and $W(t, x)$ satisfies the following.

(W1) $W(t, 0) \equiv 0$ for all $t \in R$.

(W2) $(\nabla W(t, x), x) \geq 0$, for all $(t, x) \in R \times R^{N}$.

(W3) There exist $d_{1}>0, \mu>1$ such that

$$
|\nabla W(t, x)| \leq d_{1}\left(1+|x|^{\mu}\right),
$$

for all $(t, x) \in R \times R^{N}$.

(W4) $\lim _{|x| \rightarrow 0}(\nabla W(t, x) /|x|)=0$ uniformly for $t \in R$.

(W5) There exist $v>2$ and $c>0$ such that

$$
\liminf _{|x| \rightarrow \infty} \frac{(\nabla W(t, x), x)}{|x|^{\nu}} \geq c>0
$$

uniformly for $t \in R$.

(W6) $s^{-1}(\nabla W(t, s x), x)$ is an increasing function of $s \in(0,1]$, for all $(t, x) \in R \times R^{N}$.

(W7) $W(t,-x)=W(t, x)$, for all $(t, x) \in R \times R^{N}$.

Then system (1) has infinitely many homoclinic solutions $u_{k}$ satisfying

$$
\begin{gathered}
\frac{1}{2} \int_{R}\left|\dot{u}_{k}(t)\right|^{2}+\left(L(t) u_{k}(t), u_{k}(t)\right) d t \\
-\int_{R} W\left(t, u_{k}(t)\right) d t \longrightarrow+\infty
\end{gathered}
$$

as $k \rightarrow \infty$.

In the present paper, based on the Fountain Theorem, we can prove the same result under more generic conditions, which generalizes Theorem A. Our first result can be stated as follows.
Theorem 1. Assume that $L$ satisfies $(L)$ and $\left(L^{\prime}\right)$ and $W$ satisfies (W1), (W4), (W7), (W8), and (W9).

(W8) For any $T>0$,

$$
\lim _{|x| \rightarrow \infty} \frac{W(t, x)}{|x|^{2}}=+\infty,
$$

uniformly in $t \in[-T, T]$.

(W9) there exists $\theta \geq 1$, such that

$$
\theta F(t, x) \geq F(t, s x)
$$

for all $(t, x) \in R \times R^{N}$ and $s \in[0,1]$, where $F(t, x)=$ $(\nabla W(t, x), x)-2 W(t, x)$.

Then problem (1) has infinitely many homoclinic orbits $\left\{u_{k}\right\}$ satisfying

$$
\begin{gathered}
\frac{1}{2} \int_{R}\left|\dot{u}_{k}(t)\right|^{2}+\left(L(t) u_{k}(t), u_{k}(t)\right) d t \\
-\int_{R} W\left(t, u_{k}(t)\right) d t \longrightarrow+\infty
\end{gathered}
$$

as $k \rightarrow \infty$.

Remark 2. Theorem 1 generalizes and improves Theorem A. Firstly, in Theorem 1 we remove the positiveness condition (W2) and the growth condition (W3), which are indispensable in Theorem A. Now we compare conditions (W5) and (W8), (W6), and (W9). Our condition (W8) is a local superquadratic condition and is really weaker than condition (W5). Under condition (W6), for all $s \in[0,1]$ we have

$$
\begin{aligned}
F(t, x) & -F(t, s x) \\
= & (\nabla W(t, x), x)-(\nabla W(t, s x), s x) \\
& -2[W(t, x)-W(t, s x)] \\
= & 2\left[\int_{0}^{1}(\nabla W(t, x), \tau x) d \tau-\int_{0}^{s}\left(s^{-1} \nabla W(t, s x), \tau x\right) d \tau\right] \\
& -2 \int_{s}^{1}(\nabla W(t, \tau x), x) d \tau \\
= & 2 \int_{s}^{1}\left(\nabla W(t, x)-\tau^{-1} \nabla W(t, \tau x), \tau x\right) d \tau \\
& +2 \int_{0}^{s}\left(\nabla W(t, x)-s^{-1} \nabla W(t, s x), \tau x\right) d \tau \\
\geq & 0
\end{aligned}
$$

for all $(t, x) \in R \times R^{N}$, which means that (W9) holds in the case that $\theta=1$. We consider the multiplicity of homoclinic orbits for problem (1) by using the Fountain Theorem in [27] which is simpler than the variant Fountain Theorem [26].

Moreover, under all conditions of Theorem 1 except (W7) we obtain an existence result. 
Theorem 3. Assume that $L$ satisfies $(L)$ and $\left(L^{\prime}\right)$ and $W$ satisfies (W1), (W4), (W8) and (W9). Then problem (1) possesses a nontrivial homoclinic orbit.

Remark 4. In Theorem 3, we consider the existence of homoclinic orbits for problem (1) under a class of local superquadratic conditions without the (AR) condition and any periodicity assumptions on both $L$ and $W$. There are functions $L$ and $W$ which satisfy Theorem 3, but do not satisfy the corresponding results in $[2-10,14-16,18-21,23,24]$. For example,

$$
L(t)=\left(t^{2}+1\right) I_{N}, \quad W(t, x)=2 a(t)|x|^{2} \ln \left(1+|x|^{2}\right),
$$

where $I_{N}$ is the unit matrix of order $N, a(t)=1 /\left(1+t^{2}\right)$.

\section{Preliminary Results}

In order to establish our results via critical point theory, we firstly describe some properties of the space $E$ on which the variational functional associated with problem (1) is defined. Let

$$
\begin{aligned}
E=\left\{u \in H^{1}\left(R, R^{N}\right): \int_{R}\right. & {\left[|\dot{u}(t)|^{2}\right.} \\
& +(L(t) u(t), u(t))] d t<+\infty\} .
\end{aligned}
$$

Then the space $E$ is a Hilbert space with the inner product

$$
(u, v)=\int_{R}[(\dot{u}(t), \dot{v}(t))+(L(t) u(t), v(t))] d t
$$

and the corresponding norm

$$
\|u\|^{2}=(u, u) .
$$

Note that $E \subset H^{1}\left(R, R^{N}\right) \subset L^{p}\left(R, R^{N}\right)$ for all $p \in[2,+\infty]$ with the embedding being continuous. In particular, for $p=2$ and $p=+\infty$, there exist constants $C_{2}$ and $C_{\infty}$ such that

$$
\begin{gathered}
\|u\|_{L^{2}} \leq C_{2}\|u\|, \quad \forall u \in E, \\
\|u\|_{L^{\infty}} \leq C_{\infty}\|u\|, \quad \forall u \in E .
\end{gathered}
$$

Here $L^{p}\left(R, R^{N}\right)(2 \leq p \leq+\infty)$ and $H^{1}\left(R, R^{N}\right)$ denote the Banach spaces of functions on $R$ with values in $R^{N}$ under the norms

$$
\begin{aligned}
\|u\|_{L^{p}} & =\left(\int_{R}|u(t)|^{p} d t\right)^{1 / p}, \\
\|u\|_{H^{1}} & =\left(\|u\|_{L^{2}}^{2}+\|\dot{u}\|_{L^{2}}^{2}\right)^{1 / 2},
\end{aligned}
$$

respectively. $L^{\infty}\left(R, R^{N}\right)$ is the Banach space of essentially bounded functions from $R$ into $R^{N}$ equipped with the norm

$$
\|u\|_{\infty}=\operatorname{ess} \sup \{|q(t)|: t \in R\} .
$$

Lemma 5 (see [18]). Suppose that assumption $(L)$ holds. Then the embedding of $E$ into $L^{p}\left(R, R^{N}\right)$ is compact for all $p \in$ $[2,+\infty]$.

Denote by $A$ the self-adjoint extension of the operator $-\left(d^{2} / d t^{2}\right)+L(t)$ with the domain $D(A) \subset L^{2} \equiv L^{2}\left(R, R^{N}\right)$.

Lemma 6 (see [18]). If $L$ satisfies $(L)$ and $\left(L^{\prime}\right)$, then $D(A)$ is continuously embedded in $H^{2}\left(R, R^{N}\right)$, and, consequently, one has

$$
u(t) \longrightarrow 0, \quad \dot{u}(t) \longrightarrow 0
$$

as $|t| \rightarrow \infty$, for all $u \in D(A)$.

Lemma 7. Suppose that assumptions (W1), (W4) and (W9) hold. Then $W(t, x) \geq 0$ for all $t \in R$ and $x \in R^{N}$.

Proof. Given $t \in R$ and $x \in R^{N}$, let

$$
f(s):=\frac{W(t, s x)}{s^{2}}
$$

for $s>0$; then

$$
f^{\prime}(s)=\frac{(\nabla W(t, s x), s x)-2 W(t, s x)}{s^{3}} .
$$

By (W1) and (W9), we have

$$
(\nabla W(t, x), x)-2 W(t, x) \geq 0
$$

for all $t \in R$ and $x \in R^{N}$. Hence,

$$
f^{\prime}(s) \geq 0
$$

for all $s>0$, which shows that $f(s)$ is nondecreasing in $(0,+\infty)$. It is clear that

$$
\begin{aligned}
\lim _{s \rightarrow 0} f(s) & =\lim _{s \rightarrow 0} \frac{W(t, s x)}{s^{2}} \\
& =\lim _{s \rightarrow 0} \frac{(\nabla W(t, s x), x)}{2 s} .
\end{aligned}
$$

On the other hand, by (W4) one has

$$
\lim _{s \rightarrow 0}\left|\frac{(\nabla W(t, s x), x)}{2 s}\right| \leq \lim _{s \rightarrow 0} \frac{|\nabla W(t, s x)|}{2|s x|}|x|^{2} \longrightarrow 0 .
$$

Therefore

$$
\lim _{s \rightarrow 0} f(s)=0 .
$$

Now we get $f(s) \geq 0$ for all $s>0$, which implies that

$$
W(t, x)=f(1) \geq 0
$$

for all $t \in R$ and $x \in R^{N}$.

Lemma 8. Assume that assumptions (L) and (W4) hold and $u_{n} \rightarrow u$ (weakly) in E. Then $\nabla W\left(t, u_{n}\right) \rightarrow \nabla W(t, u)$ in $L^{2}\left(R, R^{N}\right)$. 
Proof. Assume that $u_{n} \rightarrow u$ in $E$. Then there exists a constant $d>0$ such that

$$
\sup _{n \in N}\left\|u_{n}\right\|_{L^{\infty}} \leq d, \quad\|u\|_{L^{\infty}} \leq d .
$$

By (W4), for every $\varepsilon>0$, there exists $\delta>0$ such that

$$
|\nabla W(t, x)|<\varepsilon|x|
$$

for all $t \in R$ and $x \in R^{N}$ with $|x|<\delta$. Now we claim that given $d>0$, for any $\delta>0$, there exists $T_{0}>0$ such that

$$
|u(t)|<\delta
$$

for all $|t|>T_{0}$ and all $u \in E$ with $\|u\| \leq d$. If not, there exists $\delta_{0}>0$, for all $n \in N$, and there exists $u_{n} \in E$ with $\left\|u_{n}\right\| \leq d$ and $t_{n}>n$ such that

$$
\left|u_{n}\left(t_{n}\right)\right| \geq \delta_{0}
$$

On the other hand, by Lemma 5

$$
u_{n} \longrightarrow u
$$

as $n \rightarrow \infty$ in $L^{\infty}$. In view of (32) and (33), we have

$$
\begin{aligned}
\left|u\left(t_{n}\right)\right| & \geq\left|u_{n}\left(t_{n}\right)\right|-\left\|u_{n}-u\right\|_{L^{\infty}} \\
& \geq \delta_{0}-\left\|u_{n}-u\right\|_{L^{\infty}} \\
& \geq \frac{1}{2} \delta_{0}
\end{aligned}
$$

when $n$ is large enough, which is a contradiction to the fact that

$$
\lim _{|t| \rightarrow \infty}|u(t)|=0
$$

Hence, (31) holds. It follows from (29), (30), and (31) that

$$
\begin{gathered}
\left|\nabla W\left(t, u_{n}(t)\right)\right| \leq \varepsilon\left|u_{n}(t)\right| \\
|\nabla W(t, u(t))| \leq \varepsilon|u(t)|
\end{gathered}
$$

for all $n \in N$ and $|t|>T_{0}$. By Lemma $5, u_{n} \rightarrow u$ in $L^{2}\left(R, R^{N}\right)$, and $u_{n}(t) \rightarrow u(t)$ for almost every $t \in R$ and passing to a subsequence if necessary:

$$
\sum_{n=1}^{\infty}\left\|u_{n}-u\right\|_{L^{2}}<\infty
$$

which implies

$$
v_{k}(t):=\sum_{n=1}^{k}\left|u_{n}(t)-u(t)\right| \in L^{2}(R, R)
$$

for $k \in N^{+}$and

$$
\left\|v_{k_{1}}-v_{k_{2}}\right\|_{L^{2}} \leq \sum_{n=k_{1}}^{k_{2}}\left\|u_{n}-u\right\|_{L^{2}}
$$

for $k_{2}>k_{1} \in N^{+}$. Since $\left\{u_{n}\right\}$ is a Cauchy sequence in $L^{2}\left(R, R^{N}\right)$, so by (39) we know that $\left\{v_{k}\right\}$ is also a Cauchy sequence in $L^{2}(R, R)$, which together with (38) and the completeness of $L^{2}(R, R)$ shows that

$$
v(t):=\sum_{n=1}^{\infty}\left|u_{n}(t)-u(t)\right|
$$

is well defined and

$$
v \in L^{2}(R, R)
$$

In consequence,

$$
\begin{aligned}
& \left|\nabla W\left(t, u_{n}(t)\right)-\nabla W(t, u(t))\right| \\
& \quad \leq \varepsilon\left(\left|u_{n}(t)\right|+|u(t)|\right) \\
& \quad \leq \varepsilon\left(\left|u_{n}(t)-u(t)\right|+2|u(t)|\right) \\
& \quad \leq \varepsilon(v(t)+2|u(t)|)
\end{aligned}
$$

for all $n \in N$ and $|t|>T_{0}$. Consequently,

$$
\begin{aligned}
\int_{R}\left|\nabla W\left(t, u_{n}(t)\right)-\nabla W(t, u(t))\right|^{2} d t \\
=\int_{|t| \leq T_{0}}\left|\nabla W\left(t, u_{n}(t)\right)-\nabla W(t, u(t))\right|^{2} d t \\
\quad+\int_{|t|>T_{0}}\left|\nabla W\left(t, u_{n}(t)\right)-\nabla W(t, u(t))\right|^{2} d t \\
\leq \int_{|t| \leq T_{0}}\left(\left|\nabla W\left(t, u_{n}(t)\right)\right|+|\nabla W(t, u(t))|\right)^{2} d t \\
\quad+\int_{|t|>T_{0}} \varepsilon^{2}\left(|v(t)|^{2}+4|v(t)||u(t)|+4|u(t)|^{2}\right) d t \\
\leq \int_{|t| \leq T_{0}}\left(\max _{|x| \leq d}|\nabla W(t, x)|+\max _{|x| \leq d}|\nabla W(t, x)|\right)^{2} d t \\
\quad+\varepsilon^{2}\left(\|v\|_{L^{2}}^{2}+4\|v\|_{L^{2}}\|u\|_{L^{2}}+4\|u\|_{L^{2}}^{2}\right) \\
<\infty
\end{aligned}
$$

for all $n \in N$. Then using Lebesgue's convergence theorem, the lemma is proved.

In our paper we will also use the following lemma which is a special case of Lemma 1.1 in [28], due to Arioli and Szulkin [29].

Lemma 9 (see $[28,29]$ ). Let $\left\{u_{n}\right\}$ be a bounded sequence in $L^{s}\left(R, R^{N}\right), 1 \leq s<\infty$ such that $\left\{\dot{u}_{n}\right\}$ is bounded in $L^{q}\left(R, R^{N}\right)$, $1 \leq q<\infty$. If, in addition, there exists $l>0$ such that

$$
\sup _{y \in R} \int_{y-l}^{y+l}\left|u_{n}(t)\right|^{s} d t \longrightarrow 0
$$

as $n \rightarrow \infty$, then

$$
u_{n} \longrightarrow 0
$$

in $L^{p}\left(R, R^{N}\right)$ for all $p \in(s, \infty)$. 
Now we introduce some notations and some necessary definitions which will be used later. Let $B$ be a real Banach space, $I \in C^{1}(B, R)$, which means that $I$ is continuously Frechet-differentiable functional defined on B. Recall that $I \in$ $C^{1}(B, R)$ is said to satisfy the (PS) condition if any sequence $\left\{u_{n}\right\}_{n \in N} \subset B$, for which $\left\{I\left(u_{n}\right)\right\}$ is bounded and $I^{\prime}\left(u_{n}\right) \rightarrow 0$ as $n \rightarrow+\infty$ possesses a convergent subsequence in $B$.

Moreover, let $B_{r}$ be the open ball in $B$ with the radius $r$ and centered at 0 , and $\partial B_{r}$ denotes its boundary; we obtain the existence of homoclinic orbits of problem (1) by the use of the following well-known Mountain Pass Theorem [30].

Lemma 10 (see [30]). Let $B$ be a real Banach space and let $I \epsilon$ $C^{1}(B, R)$ satisfying the $(P S)$ condition. Suppose that $I(0)=0$ and that

(A1) there are constants $\rho, \alpha>0$ such that $\left.I\right|_{\partial B_{\rho}} \geq \alpha$,

(A2) there is an $e \in B \backslash \bar{B}_{\rho}$ such that $I(e)<0$.

Then I possesses a critical value $c \geq \alpha$. Moreover $c$ can be characterized as

$$
c=\inf _{f \in \Gamma} \max _{t \in[0,1]} I(g(t)),
$$

where

$$
\Gamma=\{f \in C([0,1], B): f(0)=0, f(1)=e\} .
$$

As shown in [31], a deformation lemma can be proved with the $(C)_{c}$ condition replacing the usual (PS) condition, and it turns out that Lemma 10 holds true under the $(C)_{c}$ condition.

In order to prove the multiplicity of homoclinic orbits, we will use the Fountain Theorem. Since $E$ is a Hilbert space, then there exists a basis $\left\{v_{n}\right\} \subset X$ such that $X=\overline{\oplus_{j \geq 1} X_{j}}$, where $X_{j}=\operatorname{span}\left\{v_{j}\right\}$. Letting $Y_{k}=\oplus_{j=1}^{k} X_{j}, Z_{k}=\overline{\oplus_{j \geq k} X_{j}}$, now we show the following Fountain Theorem.

Lemma 11 (see [27]). If $I \in C^{1}(X, R)$ satisfies the $(C)_{c}$ condition, $I(-u)=I(u)$, and for every $k \in N$, there exists $\rho_{k}>r_{k}>0$ such that

(i) $b_{k}:=\inf _{u \in Z_{k},\|u\|=r_{k}} I(u) \rightarrow+\infty$, as $k \rightarrow+\infty$;

(ii) $a_{k}:=\max _{u \in Y_{k},\|u\|=\rho_{k}} I(u) \leq 0$.

Then I has a sequence of critical points $\left\{u_{k}\right\}$ such that $I\left(u_{k}\right) \rightarrow$ $+\infty$ as $k \rightarrow \infty$.

In the proof of Theorem 1, the following lemma will also be used. A similar result with respect to elliptic problem has been proved in [27].

Lemma 12. Suppose that $2 \leq p \leq+\infty$; then one has

$$
\beta_{k}(p):=\sup _{u \in Z_{k},\|u\|=1}\|u\|_{L^{p}} \longrightarrow 0
$$

as $k \rightarrow \infty$.
Proof. It is clear that $0<\beta_{k+1}(p) \leq \beta_{k}(p)$, so there exists $\beta(p)$ such that

$$
\beta_{k}(p) \longrightarrow \beta(p) \geq 0
$$

as $k \rightarrow \infty$ for every $2 \leq p \leq+\infty$. By the definition of $\beta_{k}(p)$, there exists $u_{k}(p) \in Z_{k}$ with $\left\|u_{k}(p)\right\|=1$ such that

$$
\left\|u_{k}(p)\right\|_{L^{p}}>\frac{\beta_{k}(p)}{2}
$$

for every $2 \leq p \leq+\infty$ and $k \in N^{+}$. Since $\left\{u_{k}(p)\right\}_{k \in N}$ is bounded, then there exists $u(p) \in E$ such that

$$
u_{k}(p) \rightarrow u(p)
$$

as $k \rightarrow \infty$. Now since $\left\{v_{j}\right\}$ is a basis of $E$, it follows that for all $j \in N$

$$
\begin{aligned}
0 & =\left(u_{k}(p), v_{j}\right) \quad \forall k>j \\
& \longrightarrow\left(u(p), v_{j}\right)
\end{aligned}
$$

as $k \rightarrow \infty$, which shows that $u(p)=0$. By Lemma 5 we have

$$
u_{k}(p) \longrightarrow 0
$$

in $L^{p}$ for all $2 \leq p \leq+\infty$, which together with (49) and (50) implies that $\beta(p)=0$.

\section{Proof of Theorems}

Define the functional $I: E \rightarrow R$ by

$$
\begin{aligned}
I(u) & =\int_{R}\left[\frac{1}{2}|\dot{u}(t)|^{2}+\frac{1}{2}(L(t) u(t), u(t))-W(t, u(t))\right] d t \\
& =\frac{1}{2}\|u\|^{2}-\int_{R} W(t, u(t)) d t
\end{aligned}
$$

Lemma 13. Under the conditions (L), ( $\left.L^{\prime}\right)$, and (W4), $I \in$ $C^{1}(E, R)$, and for all $u, v \in E$ one has

$$
\begin{gathered}
\left\langle I^{\prime}(u), v\right\rangle=\int_{R}[(\dot{u}(t), \dot{v}(t))+(L(t) u(t), v(t)) \\
-(\nabla W(t, u(t)), v(t))] d t .
\end{gathered}
$$

Moreover, any critical point of I on $E$ is a solution of problem (1) with $u( \pm \infty)=0$ and $\dot{u}( \pm \infty)=0$.

Proof. We firstly show that $I$ is well defined. It follows from (30) that for any $\varepsilon>0$, there exists $\delta>0$ such that

$$
0 \leq W(t, x) \leq \frac{1}{2} \varepsilon|x|^{2}
$$

for all $t \in R$ and $x \in R^{N}$ with $|x|<\delta$. Letting $u \in E$, then $u \in C^{0}\left(R, R^{N}\right)$, the space of continuous function $u$ on $R$, such 
that $u(t) \rightarrow 0$ as $|t| \rightarrow \infty$. Therefore there exists $T_{1}>0$ such that

$$
|u(t)|<\delta
$$

for all $|t|>T_{1}$. Hence, one has

$$
\begin{aligned}
\int_{R} W(t, u(t)) d t & \leq \int_{-T_{1}}^{T_{1}} W(t, u(t)) d t+\frac{1}{2} \varepsilon \int_{|t|>T_{1}}|u(t)|^{2} d t \\
& \leq \int_{-T_{1}}^{T_{1}} W(t, u(t)) d t+\frac{1}{2} \varepsilon\|u\|_{L^{2}}^{2} \\
& <\infty
\end{aligned}
$$

so $I$ is well defined.

Next we prove that $I \in C^{1}(E, R)$. Rewrite $I$ as follows

$$
I=I_{1}-I_{2}
$$

where

$$
\begin{gathered}
I_{1}=\int_{R}\left[\frac{1}{2}|\dot{u}(t)|^{2}+\frac{1}{2}(L(t) u(t), u(t))\right] d t, \\
I_{2}=\int_{R} W(t, u(t)) d t .
\end{gathered}
$$

It is easy to check that $I_{1} \in C^{1}(E, R)$ and

$$
\left\langle I_{1}^{\prime}(u), v\right\rangle=\int_{R}[(\dot{u}(t), \dot{v}(t))+(L(t) u(t), v(t))] d t .
$$

It remains to show that $I_{2} \in C^{1}(E, R)$. By the mean value theorem, for any $u, v \in E$ and $h \in[0,1]$ we have

$$
\begin{aligned}
W & (t, u(t)+h v(t))-W(t, u(t)) \\
& =(\nabla W(t, u(t)+h \theta(t) v(t)), v(t)),
\end{aligned}
$$

where $\theta(t) \in(0,1)$. For any $u, v \in E$, there exists $T_{2}>0$ such that

$$
|u(t)|+|v(t)|<\delta
$$

for all $|t|>T_{2}$, so that

$$
|u(t)+h \theta(t) v(t)|<\delta
$$

for all $|t|>T_{2}$, which together with (15) and (30) implies

$$
\begin{aligned}
\int_{R} \max _{h \in[0,1]}|(\nabla W(t, u(t)+h \theta(t) v(t)), v(t))| d t \\
=\int_{|t| \leq T_{2}} \max _{h \in[0,1]}|(\nabla W(t, u(t)+h \theta(t) v(t)), v(t))| d t \\
\quad+\int_{|t|>T_{2}} \max _{h \in[0,1]}|(\nabla W(t, u(t)+h \theta(t) v(t)), v(t))| d t \\
\leq \int_{|t| \leq T_{2}|x| \leq\|u\|_{L^{\infty}}+\|v\|_{L^{\infty}}}|\nabla W(t, x)||v(t)| d t \\
\quad+\int_{|t|>T_{2}} \varepsilon\left[|u(t)||v(t)||v(t)|^{2}\right] d t \\
\leq \int_{|t| \leq T_{2}|x| \leq\|u\|_{L^{\infty}}+\|v\|_{L^{\infty}}}|\nabla W(t, x)||v(t)| d t \\
\quad+\varepsilon\left(\|u\|_{L^{2}}\|v\|_{L^{2}}+\|v\|_{L^{2}}^{2}\right) \\
<\infty .
\end{aligned}
$$

Then by Lebesgue's convergence theorem, we have

$$
\begin{aligned}
& \left\langle I_{2}^{\prime}(u), v\right\rangle \\
& \quad=\lim _{h \rightarrow 0^{+}} \frac{I_{2}(u+h v)-I_{2}(u)}{h} \\
& \quad=\lim _{h \rightarrow 0^{+}} \int_{R}[W(t, u(t)+h v(t))-W(t, u(t))] d t \\
& =\lim _{h \rightarrow 0^{+}} \int_{R}(\nabla W(t, u(t)+h \theta(t) v(t)), v(t)) d t \\
& =\int_{R}(\nabla W(t, u(t)), v(t)) d t .
\end{aligned}
$$

Now we show that $I_{2}^{\prime}$ is continuous. Supposing that $u_{n} \rightarrow u$ in $E$, by an easy computation, one has

$$
\begin{aligned}
\sup _{\|v\|=1} & \left|\left\langle I_{2}^{\prime}\left(u_{n}\right)-I_{2}^{\prime}(u), v\right\rangle\right| \\
& =\sup _{\|v\|=1}\left|\int_{R}\left(\nabla W\left(t, u_{n}(t)\right)-\nabla W(t, u(t)), v(t)\right) d t\right| \\
& \leq \sup _{\|v\|=1}\left\|\nabla W\left(t, u_{n}(t)\right)-\nabla W(t, u(t))\right\|_{L^{2}}\|v\|_{L^{2}} \\
& \leq C_{2}\left\|\nabla W\left(t, u_{n}(t)\right)-\nabla W(t, u(t))\right\|_{L^{2}} .
\end{aligned}
$$

Hence by Lemma 8, we obtain

$$
\left\langle I_{2}^{\prime}\left(u_{n}\right)-I_{2}^{\prime}(u), v\right\rangle \longrightarrow 0
$$

as $n \rightarrow \infty$ uniformly with respect to $v$, which implies the continuity of $I_{2}^{\prime}$. Now we have proved

$$
I \in C^{1}(E, R) .
$$


Finally, we show that any critical point $u$ of $I$ is a solution of problem (1) satisfying $u( \pm \infty)=0$ and $\dot{u}( \pm \infty)=0$. If $u \in E$ is a critical point of $I$, a standard argument shows that $u \in$ $C^{2}\left(R, R^{N}\right)$ satisfies (1). By Lemma 6, we only need to show that $u$ is an element of $D(A)$. It follows from (30) and (57) that

$$
|\nabla W(t, u(t))|<\varepsilon|u(t)|
$$

for all $|t|>T_{1}$. Hence, one has

$$
\begin{aligned}
\int_{R}|\nabla W(t, u(t))|^{2} d t \\
\quad \leq \int_{-T_{1}}^{T_{1}}|\nabla W(t, u(t))|^{2} d t+\varepsilon^{2} \int_{|t|>T_{1}}|u(t)|^{2} d t \\
\quad \leq \int_{-T_{1}}^{T_{1}}|\nabla W(t, u(t))|^{2} d t+\varepsilon^{2}\|u\|_{L^{2}}^{2} \\
\quad<\infty
\end{aligned}
$$

so $\nabla W(t, u) \in L^{2}\left(R, R^{N}\right)$, which together with (1) implies that $A u \in L^{2}\left(R, R^{N}\right)$. This means; $u \in D(A)$, and the proof is completed.

Lemma 14. Under conditions (L), (W4), and (W9), I satisfies the $(C)_{c}$ condition.

Proof. I satisfies the $(C)_{c}$ condition; that is, for every $\left\{u_{n}\right\} \subset E$, $\left\{u_{n}\right\}$ has a convergent subsequence if $I\left(u_{n}\right)$ is bounded and $\left(1+\left\|u_{n}\right\|\right)\left\|I^{\prime}\left(u_{n}\right)\right\| \rightarrow 0$ as $n \rightarrow \infty$. Assume that $\left\{u_{n}\right\} \subset E$ is a sequence such that

$$
I\left(u_{n}\right) \longrightarrow c
$$

is bounded and

$$
\left(1+\left\|u_{n}\right\|\right)\left\|I^{\prime}\left(u_{n}\right)\right\| \longrightarrow 0
$$

as $n \rightarrow \infty$. Hence, we have

$$
\begin{gathered}
\lim _{n \rightarrow \infty} \int_{R}\left[\frac{1}{2}\left(\nabla W\left(t, u_{n}(t)\right), u_{n}(t)\right)-W\left(t, u_{n}(t)\right)\right] d t \\
\quad=\lim _{n \rightarrow \infty}\left(I\left(u_{n}\right)-\frac{1}{2}\left\langle I^{\prime}\left(u_{n}\right), u_{n}\right\rangle\right)=c .
\end{gathered}
$$

Firstly, we show that $\left\{u_{n}\right\}$ is bounded; if not, up to a subsequence we have

$$
\left\|u_{n}\right\| \longrightarrow \infty
$$

as $n \rightarrow \infty$. Letting $v_{n}=u_{n} /\left\|u_{n}\right\|$, then $\left\{v_{n}\right\}$ is bounded in $E$. By Lemma 5, we have

$$
v_{n} \rightarrow v \quad \text { in } E, \quad v_{n} \longrightarrow v \quad \text { in } L^{2}\left(R, R^{N}\right)
$$

as $n \rightarrow \infty$. We claim the following.

Claim 1. consider

$$
\lim _{n \rightarrow \infty} \sup _{y \in R} \int_{y-1}^{y+1}\left|v_{n}(t)\right|^{2} d t=0
$$

Otherwise, for some $\sigma>0$, up to a subsequence we have

$$
\sup _{y \in R} \int_{y-1}^{y+1}\left|v_{n}(t)\right|^{2} d t \geq \sigma>0 .
$$

We can choose $\left\{y_{n}\right\} \subset R$ such that

$$
\int_{y_{n}-1}^{y_{n}+1}\left|v_{n}(t)\right|^{2} d t \geq \frac{\sigma}{2}
$$

In view of $v_{n} \rightarrow v$ in $L^{2}\left(R, R^{N}\right)$ and (79), we have

$$
\begin{aligned}
\|v\|_{L^{2}}^{2}+\frac{\sigma}{4} & \geq \int_{R}|v(t)|^{2} d t+\int_{R}\left|v_{n}(t)-v(t)\right|^{2} d t \\
& \geq \int_{y_{n}-1}^{y_{n}+1}|v(t)|^{2} d t+\int_{y_{n}-1}^{y_{n}+1}\left|v_{n}(t)-v(t)\right|^{2} d t \\
& \geq \int_{y_{n}-1}^{y_{n}+1}\left|v_{n}(t)\right|^{2} d t \\
& \geq \frac{\sigma}{2}
\end{aligned}
$$

when $n$ is large enough. By (80), there exists $\varepsilon_{0}>0$, such that the set $\Theta=\left\{t \in R:|v(t)| \geq \varepsilon_{0}\right\}$ has a positive Lebesgue measure. Moreover similar to (57), there exists $T_{3}>0$ such that $|v(t)|<\varepsilon_{0}$ for all $|t|>T_{3}$, which implies that $\Theta \subset$ $\left[-T_{3}, T_{3}\right]$. For all $t \in \Theta$, one has $\left|u_{n}(t)\right| \rightarrow \infty$ as $n \rightarrow \infty$, which together with (W8) shows

$$
\frac{W\left(t, u_{n}(t)\right)}{\left\|u_{n}(t)\right\|^{2}}=\frac{W\left(t, u_{n}(t)\right)}{\left|u_{n}(t)\right|^{2}}\left|v_{n}(t)\right|^{2} \longrightarrow+\infty
$$

as $n \rightarrow \infty$ uniformly for all $t \in \Theta$. Hence by Lemma 6 and the fact that $W(t, x) \geq 0$ for all $t \in R$ and $x \in R^{N}$, we have

$$
\begin{aligned}
\frac{1}{2}-\frac{c+o(1)}{\left\|u_{n}\right\|^{2}} & =\frac{(1 / 2)\left\|u_{n}\right\|^{2}-I\left(u_{n}\right)}{\left\|u_{n}\right\|^{2}} \\
& =\int_{R} \frac{W\left(t, u_{n}(t)\right)}{\left\|u_{n}\right\|^{2}} d t \\
& =\int_{R} \frac{W\left(t, u_{n}(t)\right)}{\left|u_{n}(t)\right|^{2}}\left|v_{n}(t)\right|^{2} d t \\
& \geq \int_{\Theta} \frac{W\left(t, u_{n}(t)\right)}{\left|u_{n}(t)\right|^{2}}\left|v_{n}(t)\right|^{2} d t \\
& \longrightarrow \infty
\end{aligned}
$$

as $n \rightarrow \infty$, which is a contradiction. Therefore we have proved Claim 1. Since $\left\|v_{n}\right\|$ is bounded, by Lemma 9 , we have

$$
v_{n} \longrightarrow 0
$$

in $L^{q}\left(R, R^{N}\right)$ for all $q>2$. Next, we will derive a contradiction. For any given $r>0,\left\|r v_{n}\right\|=r$. Similar to (31), for $\delta>0$ defined in (56), there exists $T_{4}>0$ such that

$$
\left|r v_{n}(t)\right|<\delta
$$


for all $|t|>T_{4}$ and all $n \in N$, which together with (56) shows that

$$
0 \leq W\left(t, r v_{n}(t)\right) \leq \frac{\varepsilon}{2} r^{2}\left|v_{n}(t)\right|^{2}
$$

for all $n \in N$ and $|t|>T_{4}$. In view of (83), $v_{n} \rightarrow 0$ in $L^{3}\left(R, R^{N}\right)$, which implies that

$$
\begin{aligned}
& \int_{|t| \leq T_{4}}\left|v_{n}(t)\right|^{2} d t \\
& \quad=\left(\int_{|t| \leq T_{4}} 1 d t\right)^{1 / 3}\left(\int_{|t| \leq T_{4}}\left|v_{n}(t)\right|^{3} d t\right)^{2 / 3} \longrightarrow 0
\end{aligned}
$$

as $n \rightarrow \infty$. We can derive from (W4) that

$$
\max _{|x| \leq r C_{\infty}} \frac{|\nabla W(t, x)|}{|x|}
$$

is bounded for all $|t| \leq T_{4}$. Combining (86) and (87), we have

$$
\int_{|t| \leq T_{4}} \max _{|x| \leq r C_{\infty}} \frac{|\nabla W(t, x)|}{|x|}\left|v_{n}(t)\right|^{2} d t<\varepsilon
$$

when $n$ is large enough. It follows from (85) and (88) that

$$
\begin{aligned}
0 \leq & \int_{R} W\left(t, r v_{n}(t)\right) d t \\
= & \int_{|t|>T_{4}} W\left(t, r v_{n}(t)\right) d t+\int_{|t| \leq T_{4}} W\left(t, r v_{n}(t)\right) d t \\
= & \int_{|t|>T_{4}} \frac{\varepsilon}{2} r^{2}\left|v_{n}(t)\right|^{2} d t \\
& +\int_{|t| \leq T_{4}} \int_{0}^{1}\left(\nabla W\left(t, s r v_{n}(t)\right), r v_{n}(t)\right) d s d t \\
= & \int_{|t|>T_{4}} \frac{\varepsilon}{2} r^{2}\left|v_{n}(t)\right|^{2} d t \\
& +\int_{|t| \leq T_{4}} \int_{0}^{1} \frac{\left|\nabla W\left(t, s r v_{n}(t)\right)\right|}{\left|s r v_{n}(t)\right|}\left|r v_{n}(t)\right|^{2} s d s d t \\
\leq & \int_{R} \frac{\varepsilon}{2} r^{2}\left|v_{n}(t)\right|^{2} d t \\
& +\int_{|t| \leq T_{4}} \frac{r^{2}}{2} \max _{|x| \leq r C_{\infty}} \frac{|\nabla W(t, x)|}{|x|}\left|v_{n}(t)\right|^{2} d t \\
\leq & \frac{\varepsilon}{2} r^{2} C_{2}^{2}+\frac{r^{2}}{2} \varepsilon
\end{aligned}
$$

when $n$ is large enough, which implies that

$$
\lim _{n \rightarrow \infty} \int_{R} W\left(t, r v_{n}(t)\right) d t=0
$$

for any given $r>0$. Choose a sequence $\left\{r_{n}\right\} \subset[0,1]$, such that

$$
I\left(r_{n} u_{n}\right)=\max _{r \in[0,1]} I\left(r u_{n}\right) .
$$

Given $M>0$, since $n$ is large enough, we have $2 \sqrt{M}\left\|u_{n}\right\|^{-1} \epsilon$ $[0,1]$; using (90) with $r=2 \sqrt{M}$, we obtain

$$
\begin{aligned}
I\left(r_{n} u_{n}\right) & \geq I\left(\frac{2 \sqrt{M}}{\left\|u_{n}\right\|} u_{n}\right) \\
& =I\left(2 \sqrt{M} v_{n}\right) \\
& =2 M-\int_{R} W\left(t, 2 \sqrt{M} v_{n}\right) d t \\
& \geq M
\end{aligned}
$$

for $n$ large enough, which together with the arbitrary of $M$ implies that

$$
I\left(r_{n} u_{n}\right) \longrightarrow+\infty
$$

as $n \rightarrow \infty$. In view of $(91)$ and the fact that $r_{n} \in(0,1)$, we have

$$
\begin{aligned}
& \int_{R}\left[\left|r_{n} \dot{u}_{n}\right|^{2}+\left(L(t) r_{n} u_{n}, r_{n} u_{n}\right)-\left(\nabla W\left(t, r_{n} u_{n}\right), r_{n} u_{n}\right)\right] d t \\
& \quad=\left\langle I^{\prime}\left(r_{n} u_{n}\right), r_{n} u_{n}\right\rangle \\
& \quad=\left.r_{n} \frac{d}{d t}\right|_{r=r_{n}} I\left(r u_{n}\right) \\
& \quad=0 .
\end{aligned}
$$

By (W9), we get

$$
\int_{R} F\left(t, u_{n}\right) d t \geq \frac{1}{\theta} \int_{R} F\left(t, r_{n} u_{n}\right) d t
$$

for all $t \in R$ and $n \in N$. It follows from (93) and (94) that

$$
\begin{aligned}
\int_{R}\left[\frac{1}{2}\left(\nabla W\left(t, u_{n}\right), u_{n}\right)-W\left(t, u_{n}\right)\right] d t \\
\geq \frac{1}{\theta} \int_{R}\left[\frac{1}{2}\left(\nabla W\left(t, r_{n} u_{n}\right), r_{n} u_{n}\right)-W\left(t, r_{n} u_{n}\right)\right] d t \\
=\frac{1}{\theta} \int_{R}\left[\frac{1}{2}\left(\left|r_{n} \dot{u}_{n}\right|^{2}+\left(L(t) r_{n} u_{n}, r_{n} u_{n}\right)\right)\right. \\
\left.\quad-W\left(t, r_{n} u_{n}\right)\right] d t \\
=\frac{1}{\theta} I\left(r_{n} u_{n}\right) \\
\longrightarrow+\infty
\end{aligned}
$$

as $n \rightarrow \infty$, which contradicts (74). Therefore we have proved that $\left\{u_{n}\right\}$ is bounded.

By Lemma 5 and the fact that $\left\{u_{n}\right\}$ is bounded in $E$, there exist $u \in E, d>0$, and a subsequence of $\left\{u_{n}\right\}$ again denoted by $\left\{u_{n}\right\}$ such that

$$
\sup _{n \in N}\left\|u_{n}\right\|_{L^{\infty}} \leq d \quad\|u\| \leq d,
$$

$$
u_{n} \rightarrow u \quad \text { in } E, \quad u_{n} \longrightarrow u \quad \text { in } L^{2}\left(R, R^{N}\right)
$$


as $n \rightarrow \infty$. Arguing as in Lemma 8 , we can also define

$$
v(t):=\sum_{n=1}^{\infty}\left|u_{n}(t)-u(t)\right|
$$

and $v \in L^{2}(R, R)$. It is obvious that

$$
\left\langle I^{\prime}\left(u_{n}\right)-I^{\prime}(u), u\right\rangle \longrightarrow 0
$$

as $n \rightarrow \infty$. By $(L),(97)$, and Lemma 8 one has

$$
\begin{aligned}
\left|\int_{R}\left(\nabla W\left(t, u_{n}(t)\right)-\nabla W(t, u(t)), u_{n}(t)\right) d t\right| \\
\quad \leq\left\|\nabla W\left(t, u_{n}(t)\right)-\nabla W(t, u(t))\right\|_{L^{2}}\left\|u_{n}(t)\right\|_{L^{2}} \\
\quad \leq C_{2}\left\|\nabla W\left(t, u_{n}(t)\right)-\nabla W(t, u(t))\right\|_{L^{2}}\left\|u_{n}\right\| \\
\quad \leq C_{2} d\left\|\nabla W\left(t, u_{n}(t)\right)-\nabla W(t, u(t))\right\|_{L^{2}} \\
\quad \longrightarrow 0
\end{aligned}
$$

as $n \rightarrow \infty$, which implies that

$$
\left\langle I^{\prime}\left(u_{n}\right)-I^{\prime}(u), u_{n}\right\rangle \longrightarrow 0
$$

as $n \rightarrow \infty$. Summing up (100) and (102), we have

$$
\left\langle I^{\prime}\left(u_{n}\right)-I^{\prime}(u), u_{n}-u\right\rangle \longrightarrow 0
$$

as $n \rightarrow \infty$. On the other hand, by Lemma 7 and (97) we get

$$
\begin{aligned}
& \mid \int_{R}(\left.\nabla W\left(t, u_{n}(t)\right)-\nabla W(t, u(t)), u_{n}(t)-u(t)\right) d t \mid \\
& \quad \leq \|\left(\nabla W\left(t, u_{n}(t)\right)-\nabla W(t, u(t)) \|_{L^{2}}\right. \\
& \quad \times\left\|\left(u_{n}(t)-u(t)\right)\right\|_{L^{2}} \\
& \quad \leq C_{2}\left\|\nabla W\left(t, u_{n}(t)\right)-\nabla W(t, u(t))\right\|_{L^{2}}\left\|u_{n}-u\right\| \\
& \quad \leq 2 C_{2} d\left\|\nabla W\left(t, u_{n}(t)\right)-\nabla W(t, u(t))\right\|_{L^{2}} \\
& \quad \longrightarrow 0
\end{aligned}
$$

as $n \rightarrow \infty$. An easy computation shows that

$$
\begin{gathered}
\left\langle I^{\prime}\left(u_{n}\right)-I^{\prime}(u), u_{n}-u\right\rangle \\
=\left\|u_{n}-u\right\|^{2} \\
-\int_{R}\left(\nabla W \left(t, u_{n}(t)-\nabla W(t, u(t)),\right.\right. \\
\left.\left.u_{n}(t)-u(t)\right)\right) d t .
\end{gathered}
$$

Consequently, $\left\|u_{n}-u\right\| \rightarrow 0$ as $n \rightarrow \infty$.

Proof of Theorem 1. By Lemma 13 and Lemma 14, I $\in C^{1}$ $(E, R)$ satisfies the $(C)_{c}$ condition and $I(u)=I(-u)$; hence to prove Theorem 1 we should just show that $I$ has the geometric properties (i) and (ii) of Lemma 11. (i) By Lemma 12

$$
\beta_{k}(p)=\sup _{u \in Z_{k},\|u\|=1}\|u\|_{L^{p}} \longrightarrow 0
$$

as $k \rightarrow \infty$ for $p \in[2,+\infty]$. We choose $r_{k}=\min \left\{1 / \beta_{k}(\infty)\right.$, $\left.1 / \beta_{k}(4)\right\}$; then $r_{k} \rightarrow \infty$ as $k \rightarrow \infty$, and for every $u \in Z_{k}$ with $\|u\|=r_{k}$, we have

$$
\|u\|_{L^{\infty}} \leq \beta_{k}(\infty) r_{k}<1,\|u\|_{L^{4}} \leq \beta_{k}(4) r_{k}<1 .
$$

Similar to (31), there exists $T_{5}>0$ such that

$$
|u(t)|<\delta
$$

for all $|t|>T_{5}$ and all $u \in Z_{k}$ with $\|u\|=r_{k}$, where $\delta$ is defined in (56). Consequently, by (56), for any $\varepsilon>0$

$$
0 \leq W(t, u(t)) \leq \frac{1}{2} \varepsilon|u(t)|^{2}
$$

for all $|t|>T_{5}$ and all $u \in Z_{k}$ with $\|u\|=r_{k}$. Hence, we have for all $u \in Z_{k}$ with $\|u\|=r_{k}$

$$
\begin{aligned}
I(u) & =\frac{1}{2}\|u\|^{2}-\int_{|t|>T_{5}} W(t, u(t)) d t-\int_{|t| \leq T_{5}} W(t, u(t)) d t \\
& \geq \frac{1}{2}\|u\|^{2}-\int_{|t|>T_{5}} \frac{1}{2} \varepsilon|u(t)|^{2} d t-\int_{|t| \leq T_{5}} \max _{|x| \leq 1} W(t, x) d t \\
& \geq \frac{1}{2}\|u\|^{2}-\frac{1}{2} \varepsilon\left(\beta_{k}(2)\right)^{2}\|u\|^{2}-\int_{|t| \leq T_{5}} \max _{|x| \leq 1} W(t, x) d t \\
& \geq \frac{1}{4}\|u\|^{2}-\int_{|t| \leq T_{5}} \max _{|x| \leq 1} W(t, x) d t
\end{aligned}
$$

when $\varepsilon$ is small enough. Therefore, one has

$$
\begin{aligned}
b_{k} & =\inf _{u \in Z_{k},\|u\|=r_{k}} I(u) \\
& \geq \inf _{u \in Z_{k},\|u\|=r_{k}}\left(\frac{1}{4}\|u\|^{2}-\int_{|t| \leq T_{5}} \max _{|x| \leq 1} W(t, x) d t\right) \\
& =\frac{1}{4} r_{k}^{2}-\int_{|t| \leq T_{5}} \max _{|x| \leq 1} W(t, x) d t \\
& \longrightarrow \infty
\end{aligned}
$$

as $k \rightarrow \infty$. that

(ii) Firstly, we claim that there exists a constant $\varepsilon>0$ such

$$
\text { meas }\{t \in R:|u(t)| \geq \varepsilon\|u\|\} \geq \varepsilon
$$

for all $u \in Y_{k} \backslash\{0\}$. Otherwise, for every $n \in N$, there exists $u_{n} \in Y_{k} \backslash\{0\}$ such that

$$
\text { meas }\left\{t \in R:\left|u_{n}(t)\right| \geq \frac{1}{n}\left\|u_{n}\right\|\right\}<\frac{1}{n} \text {. }
$$

Without loss of generality, we suppose that $\left\|u_{n}\right\|=1$; then there is

$$
\text { meas }\left\{t \in R:\left|u_{n}(t)\right| \geq \frac{1}{n}\right\}<\frac{1}{n} \text {. }
$$


In view of the compactness of the unit sphere of $Y_{k}$, there exists a subsequence which is still denoted by $\left\{u_{n}\right\}$ such that $u_{n}$ converges to some $u_{0} \in Y_{k}$ as $n \rightarrow \infty$. It is clear that $\left\|u_{0}\right\|=1$. Since all the norms in $Y_{k}$ are equivalent, we have $u_{n} \rightarrow u_{0}$ in $L^{2}$ as $n \rightarrow \infty$; that is,

$$
\int_{R}\left|u_{n}(t)-u_{0}(t)\right|^{2} d t \longrightarrow 0
$$

as $n \rightarrow \infty$. Thus there exist constants $\tau_{1}$ and $\tau_{2}$ such that

$$
\text { meas }\left\{t \in R:\left|u_{0}(t)\right| \geq \tau_{1}\right\} \geq \tau_{2} \text {. }
$$

If not, we have

$$
\text { meas }\left\{t \in R:\left|u_{0}(t)\right| \geq \frac{1}{n}\right\}=0
$$

for all $n \in N$, which implies that

$$
\begin{aligned}
0 & \leq \int_{R}\left|u_{0}(t)\right|^{4} d t \leq\left\|u_{0}\right\|_{L^{\infty}}^{2}\left\|u_{0}\right\|_{L^{2}}^{2} \\
& <\frac{1}{n^{2}} C_{2}^{2}\left\|u_{0}\right\|^{2}=\frac{1}{n^{2}} C_{2}^{2} \longrightarrow 0
\end{aligned}
$$

as $n \rightarrow \infty$. Hence $u_{0}=0$, which contradicts $\left\|u_{0}\right\|=1$. Therefore (116) holds. Let $\Omega_{0}=\left\{t \in R:\left|u_{0}(t)\right| \geq \tau_{1}\right\}$, $\Omega_{n}=\left\{t \in R:\left|u_{n}(t)\right|<1 / n\right\}$, and $\Omega_{n}^{c}=R \backslash \Omega_{n}$. It follows from (114) and (116) that

$$
\begin{aligned}
\operatorname{meas} & \left(\Omega_{n} \backslash \Omega_{0}\right) \\
& =\operatorname{meas}\left(\Omega_{0} \backslash\left(\Omega_{n}^{c} \cap \Omega_{0}\right)\right) \\
& \geq \operatorname{meas}\left(\Omega_{0}\right)-\operatorname{meas}\left(\Omega_{n}^{c} \cap \Omega_{0}\right) \\
& =\tau_{2}-\frac{1}{n} .
\end{aligned}
$$

We can choose $n$ large enough, such that

$$
\tau_{1}-\frac{1}{n} \geq \frac{1}{2} \tau_{1}, \quad \tau_{2}-\frac{1}{n} \geq \frac{1}{2} \tau_{2} .
$$

Then we have

$$
\begin{aligned}
\left|u_{n}(t)-u_{0}(t)\right|^{2} & \geq|| u_{n}(t)|-| u_{0}(t) \|^{2} \\
& \geq\left(\tau_{1}-\frac{1}{n}\right)^{2} \geq \frac{1}{4} \tau_{1}^{2}
\end{aligned}
$$

for all $t \in \Omega_{n} \cap \Omega_{0}$. In consequence,

$$
\begin{aligned}
\int_{R} \mid u_{n} & (t)-\left.u_{0}(t)\right|^{2} d t \\
& \geq \int_{\Omega_{n} \cap \Omega_{0}}\left|u_{n}(t)-u_{0}(t)\right|^{2} d t \\
& \geq \frac{1}{4} \tau_{1}^{2} \cdot \operatorname{meas}\left(\Omega_{n} \cap \Omega_{0}\right) \\
& \geq \frac{1}{4} \tau_{1}^{2}\left(\tau_{2}-\frac{1}{n}\right) \\
& \geq \frac{1}{8} \tau_{1}^{2} \tau_{2} \\
& >0
\end{aligned}
$$

for all $n$ being large enough, which is a contradiction to (115). Hence (112) holds.

Similar to (31), for any $\varepsilon>0$ there exists $T_{6}>0$ such that

$$
|v(t)|<\varepsilon
$$

for all $|t|>T_{6}$ and all $v \in Y_{k}$ with $\|v\| \leq 1$. Consequently, for all $u \in Y_{k}$,

$$
\frac{|u(t)|}{\|u\|}<\varepsilon
$$

for all $|t|>T_{6}$, which implies that $\Omega_{u}:=\{t \in R:|u(t)| \geq$ $\varepsilon\|u\|\} \subset\left[-T_{6}, T_{6}\right]$ for all $u \in Y_{k}$. By (W8), there exists $G>0$ such that

$$
W(t, x) \geq \frac{1}{\varepsilon^{3}}|x|^{2}
$$

for all $x \in R^{N}$ with $|x| \geq G$ and $|t|<T_{6}$. Hence we have

$$
W(t, u(t)) \geq \frac{1}{\varepsilon^{3}}|u(t)|^{2} \geq \frac{1}{\varepsilon}\|u\|^{2}
$$

for all $t \in \Omega_{u}$ and $u \in Y_{k}$ with $\|u\| \geq G / \varepsilon$, which together with Lemma 7 implies that

$$
\begin{aligned}
\frac{I(u)}{\|u\|^{2}} & =\frac{1}{2}-\int_{R} \frac{W(t, u(t))}{\|u\|^{2}} d t \\
& \leq \frac{1}{2}-\int_{\Omega_{u}} \frac{W(t, u(t))}{\|u\|^{2}} d t \\
& =\frac{1}{2}-\frac{1}{\varepsilon} \cdot \operatorname{meas}\left(\Omega_{u}\right) \\
& \leq \frac{1}{2}-1<0
\end{aligned}
$$

for all $u \in Y_{k}$ and $\|u\| \geq G / \varepsilon$. So we can choose $\rho_{k}>$ $\max \left\{G / \varepsilon, r_{k}\right\}$; then

$$
a_{k}=\max _{u \in Y_{k},\|u\|=\rho_{k}} I(u) \leq 0 .
$$

Hence by Lemma 11, we obtain that problem (1) has infinitely many homoclinic solutions $\left\{u_{k}\right\}$ satisfying

$$
\begin{gathered}
\frac{1}{2} \int_{R}\left|\dot{u}_{k}(t)\right|^{2}+\left(L(t) u_{k}(t), u_{k}(t)\right) d t \\
-\int_{R} W\left(t, u_{k}(t)\right) d t \longrightarrow+\infty
\end{gathered}
$$

as $k \rightarrow \infty$.

Proof of Theorem 3. We divide the proof of Theorem 3 into the following three steps.

Step 1. It is clear that $I(0)=0$, and we have proved that $I \in C^{1}(E, R)$ satisfies the $(C)_{c}$ condition in Lemma 13 and Lemma 14.

Step 2. Letting $\rho=\delta / C_{\infty}$ and $\|u\|=\rho$, we have $\|u\|_{L^{\infty}} \leq$ $\delta$, where $C_{\infty}>0$ is defined in (16) and $\delta$ is defined in (56). Hence, by (56), for any $\varepsilon>0$

$$
0 \leq W(t, u(t)) \leq \frac{1}{2} \varepsilon|u(t)|^{2}
$$


for all $t \in R$ and $u \in E$ with $\|u\|=\rho$. In consequence, combining this with (54), we obtain

$$
\begin{aligned}
I(u) & =\frac{1}{2}\|u\|^{2}-\int_{R} W(t, u) d t \\
& \geq \frac{1}{2}\|u\|^{2}-\frac{\varepsilon}{2}\|u\|_{L^{2}}^{2} \\
& \geq\left(\frac{1}{2}-\frac{\varepsilon}{2} C_{2}^{2}\right)\|u\|^{2}
\end{aligned}
$$

for all $t \in R$ and $u \in E$ with $\|u\|=\rho$. Setting $\varepsilon=1 / 2 C_{2}^{2}$, the inequality (131) implies that

$$
\left.I\right|_{\partial B_{\rho}} \geq \frac{\rho^{2}}{4}:=\alpha>0 .
$$

Step 3. It remains to prove that there exists an $e \in E$ such that $\|e\|>\rho$ and $I(e) \leq 0$, where $\rho$ is defined in Step 2. By (W8), for any $T, M>0$, there exists $G>0$ such that

$$
\frac{W(t, x)}{|x|^{2}} \geq M
$$

for all $|x|>G$ and $t \in[-T, T]$. Letting $u_{0}(t)=e^{-t^{2}} e_{1}$, where $e_{1}=(1,0, \ldots, 0)$, hence $\left|u_{0}(t)\right|>e^{-4}$ for all $t \in[1,2]$. It is clear that when $r>G e^{4}$,

$$
\left|r u_{0}(t)\right|>G
$$

for all $t \in[1,2]$, which together with (133) shows that when $r>G e^{4}$

$$
\frac{W\left(t, r u_{0}(t)\right)}{\left|r u_{0}(t)\right|^{2}} \geq M
$$

for all $t \in[1,2]$. Combining (135), Lemma 7, and the fact that $\int_{1}^{2}\left|u_{0}(t)\right|^{2} d t>0$, we have

$$
\begin{aligned}
\frac{I\left(r u_{0}\right)}{r^{2}}= & \frac{1}{2}\left\|u_{0}\right\|^{2}-\int_{R} \frac{W\left(t, r u_{0}(t)\right)}{\left|r u_{0}(t)\right|^{2}}\left|u_{0}(t)\right|^{2} d t \\
= & \frac{1}{2}\left\|u_{0}\right\|^{2}-\int_{1}^{2} \frac{W\left(t, r u_{0}(t)\right)}{\left|r u_{0}(t)\right|^{2}}\left|u_{0}(t)\right|^{2} d t \\
& -\int_{R \backslash[1,2]} \frac{W\left(t, r u_{0}(t)\right)}{\left|r u_{0}(t)\right|^{2}}\left|u_{0}(t)\right|^{2} d t \\
\leq & \frac{1}{2}\left\|u_{0}\right\|^{2}-\int_{1}^{2} \frac{W\left(t, r u_{0}(t)\right)}{\left|r u_{0}(t)\right|^{2}}\left|u_{0}(t)\right|^{2} d t \\
\leq & \frac{1}{2}\left\|u_{0}\right\|^{2}-M \int_{1}^{2}\left|u_{0}(t)\right|^{2} d t \\
< & 0,
\end{aligned}
$$

when $r$ and $M$ are both large enough. by

By Lemma 10, $I$ possesses a critical value $c \geq \alpha>0$ given

$$
c=\inf _{f \in \Gamma} \max _{t \in[0,1]} I(f(t)),
$$

where

$$
\Gamma=\{f \in C([0,1], B): f(0)=0, f(1)=e\} .
$$

Hence there is a $u \in E$ such that

$$
I(u)=c, \quad I^{\prime}(u)=0 .
$$

Therefore $u$ is a nontrivial homoclinic orbit of problem (1). Theorem 3 is proved now.

\section{Acknowledgements}

The authors would like to thank the referee for valuable suggestions. This paper is supported by National Natural Science Foundation of China (no. 11071198) and Fundamental Funds for the Central Universities (XDJK2009C075).

\section{References}

[1] H. Poincaré, Les Méthods Nouvelles de la Mécanique Céleste, Gauthier-Villars, Paris, France, 1897-1899.

[2] V. Coti Zelati, I. Ekeland, and E. Séré, "A variational approach to homoclinic orbits in Hamiltonian systems," Mathematische Annalen, vol. 288, no. 1, pp. 133-160, 1990.

[3] P. L. Felmer and E. A. de Barros e Silva, "Homoclinic and periodic orbits for Hamiltonian systems," Annali della Scuola Normale Superiore di Pisa. Classe di Scienze, vol. 26, no. 2, pp. 285-301, 1998.

[4] V. Coti Zelati and P. H. Rabinowitz, "Homoclinic orbits for second order Hamiltonian systems possessing superquadratic potentials," Journal of the American Mathematical Society, vol. 4, no. 4, pp. 693-727, 1991.

[5] E. Serra, M. Tarallo, and S. Terracini, "Subharmonic solutions to second-order differential equations with periodic nonlinearities," Nonlinear Analysis: Theory, Methods \& Applications, vol. 41, no. 5-6, pp. 649-667, 2000.

[6] Y. Ding and C. Lee, "Homoclinics for asymptotically quadratic and superquadratic Hamiltonian systems," Nonlinear Analysis: Theory, Methods \& Applications, vol. 71, no. 5-6, pp. 1395-1413, 2009.

[7] R. Yuan and Z. Zhang, "Homoclinic solutions for a class of second order Hamiltonian systems," Results in Mathematics, vol. 61, no. 1-2, pp. 195-208, 2012.

[8] E. Paturel, "Multiple homoclinic orbits for a class of Hamiltonian systems," Calculus of Variations and Partial Differential Equations, vol. 12, no. 2, pp. 117-143, 2001.

[9] P. H. Rabinowitz, "Homoclinic orbits for a class of Hamiltonian systems," Proceedings of the Royal Society of Edinburgh. Section A, vol. 114, no. 1-2, pp. 33-38, 1990.

[10] M. Izydorek and J. Janczewska, "Homoclinic solutions for a class of the second order Hamiltonian systems," Journal of Differential Equations, vol. 219, no. 2, pp. 375-389, 2005.

[11] X. H. Tang and L. Xiao, "Homoclinic solutions for a class of second-order Hamiltonian systems," Nonlinear Analysis: Theory, Methods \& Applications, vol. 71, no. 3-4, pp. 1140-1152, 2009.

[12] C. O. Alves, P. C. Carrião, and O. H. Miyagaki, "Existence of homoclinic orbits for asymptotically periodic systems involving Duffing-like equation," Applied Mathematics Letters, vol. 16, no. 5, pp. 639-642, 2003. 
[13] P. C. Carrião and O. H. Miyagaki, "Existence of homoclinic solutions for a class of time-dependent Hamiltonian systems," Journal of Mathematical Analysis and Applications, vol. 230, no. 1, pp. 157-172, 1999.

[14] P. H. Rabinowitz and K. Tanaka, "Some results on connecting orbits for a class of Hamiltonian systems," Mathematische Zeitschrift, vol. 206, no. 3, pp. 473-499, 1991.

[15] W. Omana and M. Willem, "Homoclinic orbits for a class of Hamiltonian systems," Differential and Integral Equations, vol. 5, no. 5, pp. 1115-1120, 1992.

[16] X. Lv, S. Lu, and P. Yan, "Existence of homoclinic solutions for a class of second-order Hamiltonian systems," Nonlinear Analysis: Theory, Methods \& Applications, vol. 72, no. 1, pp. 390-398, 2010.

[17] Z. Zhang and R. Yuan, "Homoclinic solutions for a class of nonautonomous subquadratic second-order Hamiltonian systems," Nonlinear Analysis: Theory, Methods \& Applications, vol. 71, no. 9, pp. 4125-4130, 2009.

[18] Y. H. Ding, "Existence and multiplicity results for homoclinic solutions to a class of Hamiltonian systems," Nonlinear Analysis: Theory, Methods \& Applications, vol. 25, no. 11, pp. 1095-1113, 1995.

[19] M.-H. Yang and Z.-Q. Han, "Infinitely many homoclinic solutions for second-order Hamiltonian systems with odd nonlinearities," Nonlinear Analysis: Theory, Methods \& Applications, vol. 74, no. 7, pp. 2635-2646, 2011.

[20] Z.-Q. Ou and C.-L. Tang, "Existence of homoclinic solution for the second order Hamiltonian systems," Journal of Mathematical Analysis and Applications, vol. 291, no. 1, pp. 203-213, 2004.

[21] J. Yang and F. Zhang, "Infinitely many homoclinic orbits for the second-order Hamiltonian systems with super-quadratic potentials," Nonlinear Analysis: Real World Applications, vol. 10, no. 3, pp. 1417-1423, 2009.

[22] Q. Zhang and C. Liu, "Infinitely many homoclinic solutions for second order Hamiltonian systems," Nonlinear Analysis: Theory, Methods \& Applications, vol. 72, no. 2, pp. 894-903, 2010.

[23] P. Korman and A. C. Lazer, "Homoclinic orbits for a class of symmetric Hamiltonian systems," Electronic Journal of Differential Equations, vol. 1994, pp. 1-10, 1994.

[24] Y. Lv and C.-L. Tang, "Existence of even homoclinic orbits for second-order Hamiltonian systems," Nonlinear Analysis: Theory, Methods \& Applications, vol. 67, no. 7, pp. 2189-2198, 2007.

[25] J. Sun, H. Chen, and J. J. Nieto, "Homoclinic solutions for a class of subquadratic second-order Hamiltonian systems," Journal of Mathematical Analysis and Applications, vol. 373, no. 1, pp. $20-$ 29,2011

[26] W. Zou, "Variant fountain theorems and their applications," Manuscripta Mathematica, vol. 104, no. 3, pp. 343-358, 2001.

[27] M. Willem, Minimax Theorems, Progress in Nonlinear Differential Equations and Their Applications, 24, Birkhäuser, Boston, Mass, USA, 1996.

[28] P.-L. Lions, "The concentration-compactness principle in the calculus of variations. The locally compact case. II," Annales de l'Institut Henri Poincaré. Analyse Non Linéaire, vol. 1, no. 4, pp. 223-283, 1984.

[29] G. Arioli and A. Szulkin, "Homoclinic solutions for a class of systems of second order differential equations," Topological Methods in Nonlinear Analysis, vol. 6, no. 1, pp. 189-197, 1995.

[30] P. H. Rabinowitz, Minimax Methods in Critical Point Theory with Applications to Differential Equations, vol. 65 of CBMS Regional Conference Series in Mathematics, published for the
Conference Board of the Mathematical Sciences, Washington, DC, American Mathematical Society, Providence, RI, USA, 1986.

[31] P. Bartolo, V. Benci, and D. Fortunato, "Abstract critical point theorems and applications to some nonlinear problems with "strong" resonance at infinity", Nonlinear Analysis: Theory, Methods \& Applications, vol. 7, no. 9, pp. 981-1012, 1983. 


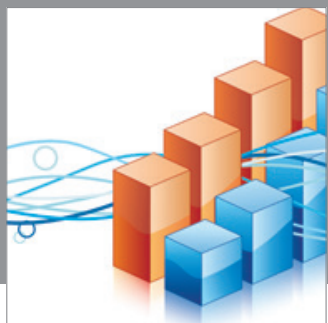

Advances in

Operations Research

mansans

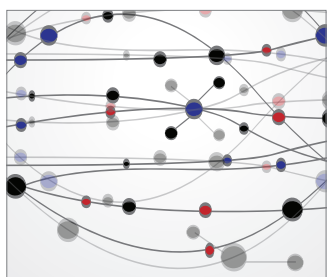

The Scientific World Journal
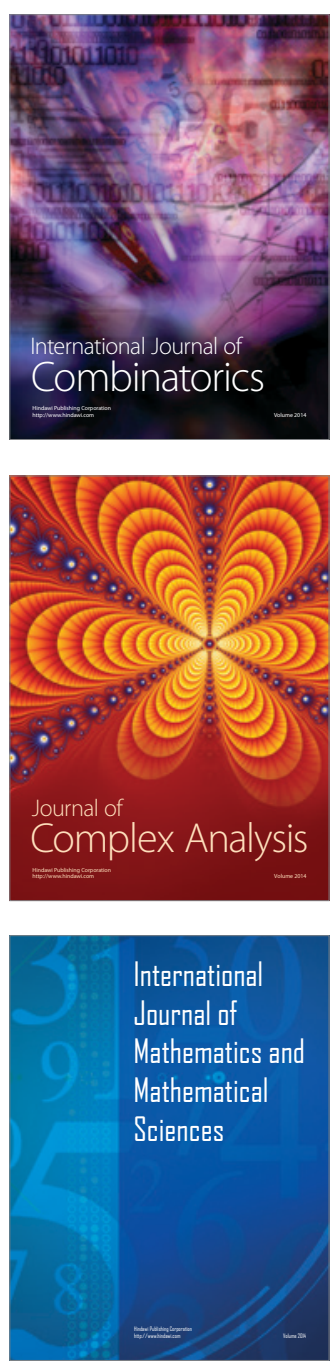
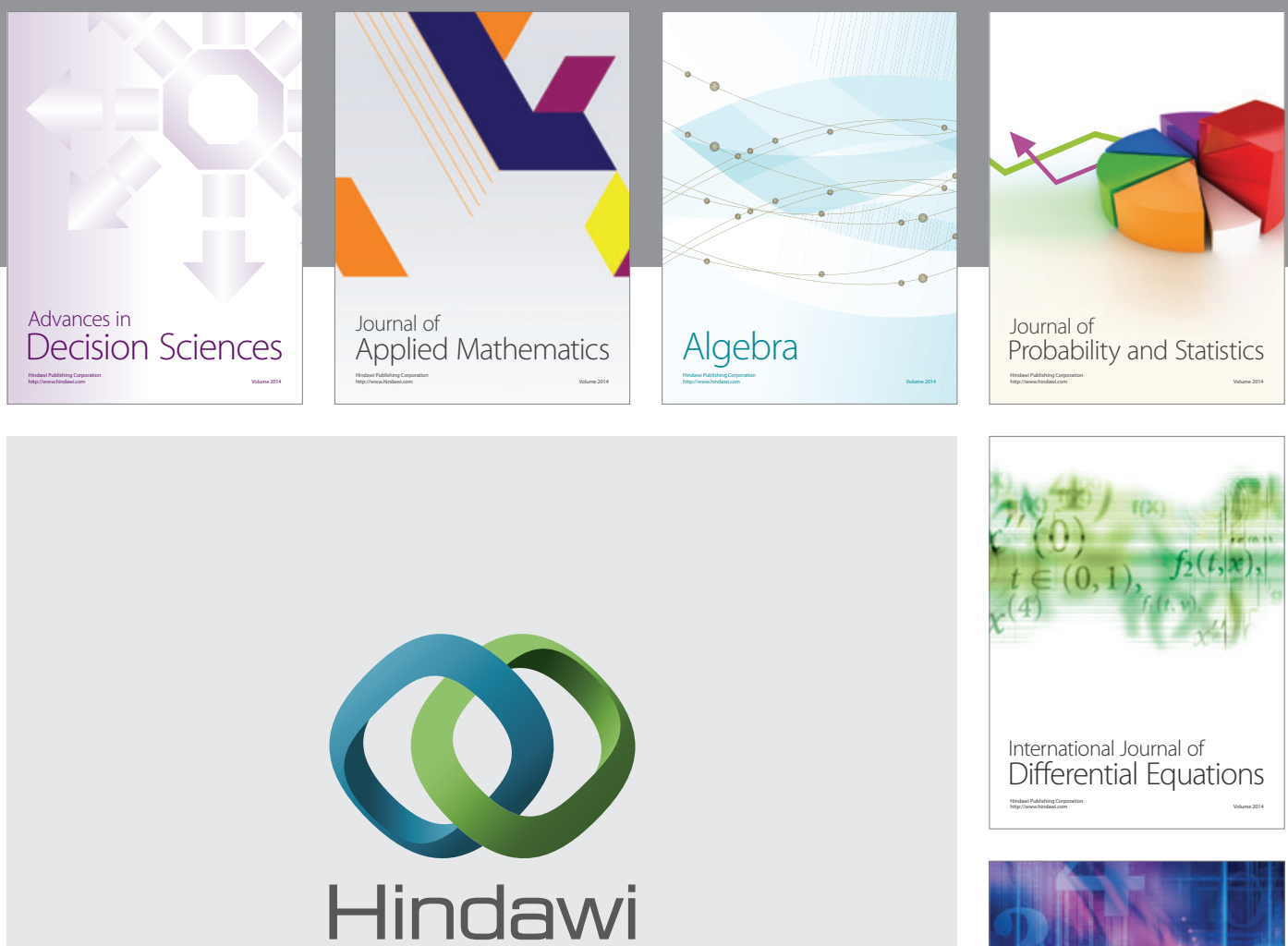

Submit your manuscripts at http://www.hindawi.com
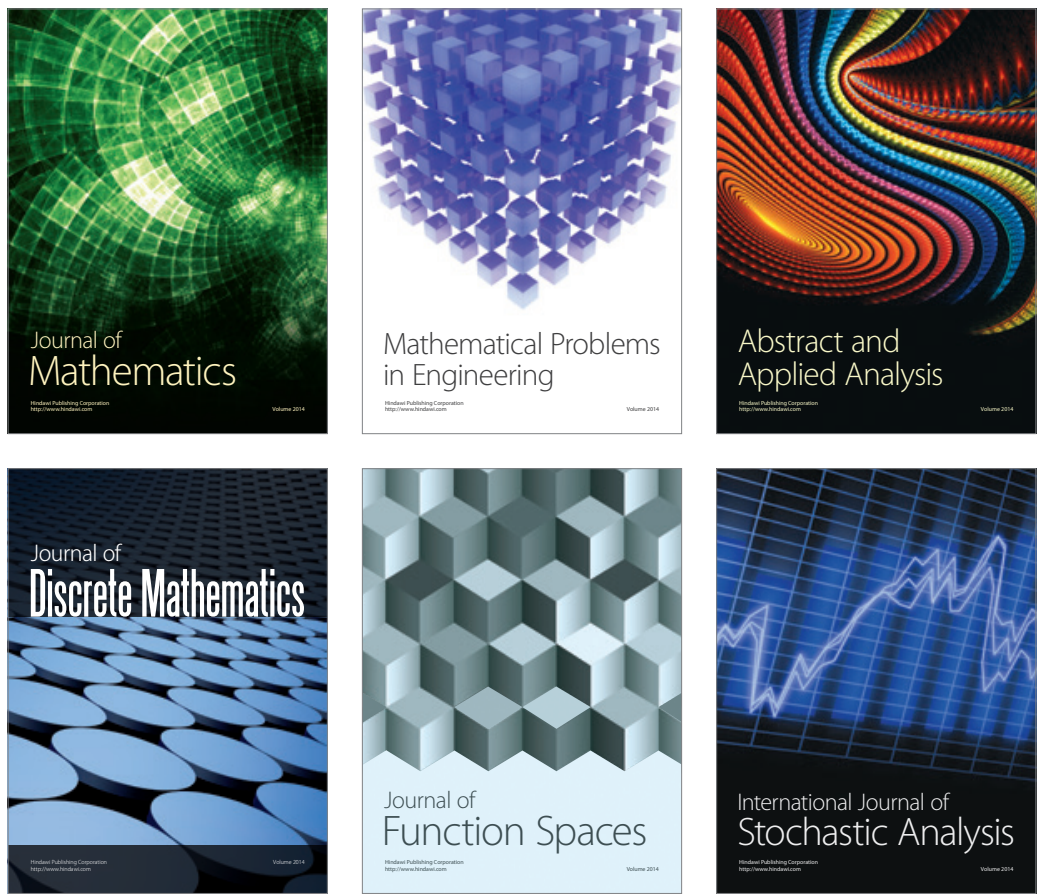

Journal of

Function Spaces

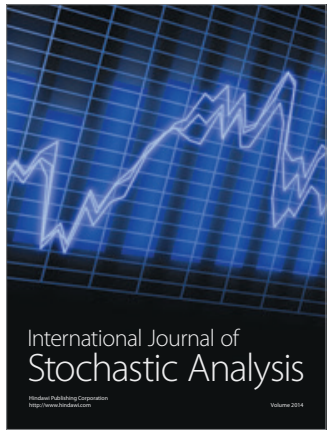

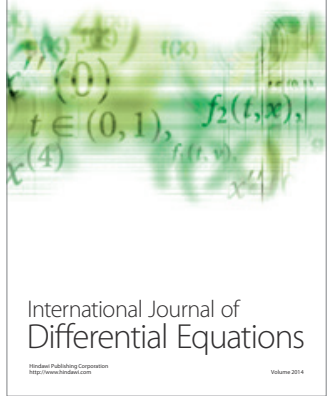
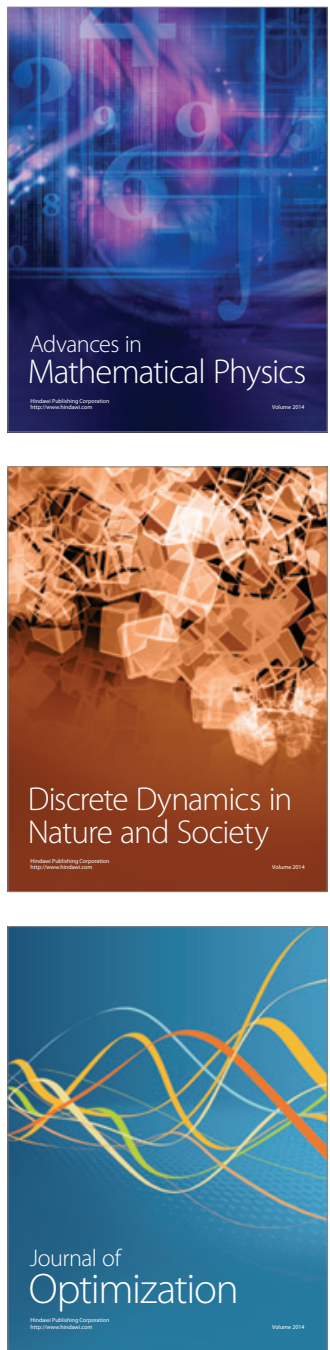